\title{
NUTRIÇÃO MINERAL DE HORTALIÇAS XV. ESTUDOS DA NUTRIÇÃO MINERAL DE DUAS VARIEDADES DE PIMENTÃO CULTIVADO EM CONDIÇÕES DE CAMPO
}

\author{
P. D. FERnANDES \\ G. D. DE Oliveira ** \\ H. P. HAAG
}

\begin{abstract}
Plantas de Pimentão (Capsicum annuum, L.), variedades Avelar e Ikeda, cultivadas em solo da série "Luiz de Queiroz", foram analisadas periodicamente, aos 30,40,60,75, 90, 100 e 115 dias de idade, para nitrogênio, fósforo, potássio, cálcio, magnésio e enxôfre. Em cada amostragem foram feitas determinações de pêso da matéria sêca, número de fôlhas e frutos e altura das plantas. Verificou-se um crescimento lento até aos 75 dias, para ambas as variedades, intensificando_se após êste período. Não se encontrou diferença significativa entre as duas variedades quanto ao crescimento. A extração de nutrientes variou paralelamente ao crescimento, sendo intensificada com a frutificação. Entre as duas variedades não ocorreu diferença significante no total de nutrientes absorvidos no final do ciclo. Porém aos 75 dias uma planta da Avelar, extraiu mais que a Ikeda: $14,7 \% \mathrm{~N}, 15,4 \% \mathrm{P}, 18,4 \% \mathrm{~K}, 16,9 \% \mathrm{Ca}, 18,1 \% \mathrm{Mg}$ e $21,8 \% \mathrm{~S}$. Nas condições do presente trabalho, uma cultura de pimentão absorveu por hectare $(25.000$ plantas $): 40,9 \mathrm{~kg} / \mathrm{N}, 3,8 \mathrm{~kg} / \mathrm{P}, 68,6 \mathrm{~kg} / \mathrm{k}, 51,8 \mathrm{~kg} / \mathrm{Ca}$, $6,7 \mathrm{~kg} / \mathrm{mg}$ e $4,3 \mathrm{~kg} / \mathrm{S}$.
\end{abstract}

\section{INTRODUÇAOO}

O pimentão (Capsicum annuum, L.) está incluído entre as hortaliças de maior consumo no Brasil. Segundo dados colhidos junto à Secretaria da Agricultura do Estado de São Paulo, em seu Instituto de Economia Agrícola - DLE SAEE, a produção de pimentão neste Estado, ano de 1969, foi da ordem de 21.400 toneladas rendendo Cr\$6.600.000,00. Em ordem de valor economico, está classificada entre as primeiras hortaliças.

Apesar da sua grande importancia, são escassas as referencias bibliográficas, enfocando sua nutrição mineral, base necessária para os estudos de adubação.

* Departamento de Agricultura e Horticultura, E.S.A. “Luiz de Queiroz" Universidade de São Paulo.

** Departamento de Química, E.S.A. "Luiz de Queiroz" - Universidade de São Paulo. 
Trabalhando com a "Var. Casca Dura", HAAG, et al (1970) relatam serem absorvidos, por uma cultura em $\mathrm{kg} / \mathrm{ha},(31.250$ plantas): $24,4 \mathrm{~N}, 6,1 \mathrm{P}, 49,0 \mathrm{~K}, 5,1 \mathrm{Ca}, 2,8 \mathrm{Mg}, 4,4 \mathrm{~S}$.

Em pesquisa conduzida, versando sobre estudos de deficiencias minerais, nas variedades Avelar e Ikeda, P. D. FERNANDES (1971), verificou uma sensibilidade maior a deficiencia dos macronutrientes em geral, na variedade Avelar.

A partir dessas observações, o presente trabalho, visa apresentar resultados de crescimento e de extração de nutrientes, por essas duas variedades, cultivadas em condições de campo.

* Revisão da Literatura

\section{MATERIAL E MÉTODOS}

Sementes de pimentão, das var. Avelar e Ikeda, foram semeadas a 14/08/1970, em sementeiras convenientemente preparadas. Germinadas a 25 desse mesmo mes, com trinta dias de idade foram transplantadas em solo da série "Luiz de Queiroz" (RANZANI et al. 1966), cujas características químicas, determinadas segundo CATANI et al. (1955), foram :

$$
\begin{aligned}
& \mathrm{pH}=6,5 \\
& \text { mat. organica } \%=2,2 \\
& \text { teor trocável } \\
& \mathrm{K}+\quad \text { e. } \mathrm{mg} \text { por } 100 \mathrm{~g} \text { de solo }=0,85 \\
& \mathrm{Ca}+\text { " " " " " }=3,80 \\
& \mathrm{Mg}++ \text { " " " " " }=1,05 \\
& \mathrm{PO}_{4}^{-3} " \text { " " " " }=0,40
\end{aligned}
$$

Usando-se um espaçamento de $1,00 \times 0,40 \mathrm{~m}$, cada planta recebeu uma adubação, segundo recomendações de BERNARDI (1962), de : $2 \mathrm{~kg}$ de esterco de curral, $150 \mathrm{~g}$ superfosfato $\left(25 \% \mathrm{P}_{2} \mathrm{O}_{5}\right), 30 \mathrm{~g}$ $\mathrm{KC} 1,45 \mathrm{~g}$ de salitre do Chile. O nitrogenio foi fracionado e aplicado aos 10, 20 e 30 dias após transplante, em cobertura. As plantas receberam os tratos culturais necessários.

Foram feitas amostragens de plantas, a partir do transplante, em intervalos variando de 10 a 20 dias. O número de plantas colhidas foi sempre igual ou superior a quatro.

Depois de colhidas, eram lavadas, separadas em raiz, caule, fôlhas e flôres/frutos, e postas a secar em estufa a $80^{\circ} \mathrm{C}$. Esse material, uma vez sêco, foi pesado e moido em micromoinho Willey.

A análise para nitrogênio, se processou pela técnica de Micro-Kjel- 
dahl, descrita em MALAVOLTA (1957); o fósforo seguiu as recomendações de LOTT et al. (1956); os elementos potássio, cálcio e 'magnésio foram determinados por espectrofotometria de absorção atômica PERKIN ELMER (1966); o enxôfre foi dosado segundo TOTH et al. (1948).

\section{RESULTADOS E DISCUSSAO}

O florescimento, tanto para a var. Avelar, como para a Ikeda, se deu em um mesmo período, aos 65 dias de idade.

O pêso da matéria sêca da planta, o número de fôlhas e frutos e a sua altura, para ambas as variedades, acham-se dispostas na Tabela $\mathrm{I}$.

TABELA I - Pêso da matéria sêca, número de fôlhas e frutos e altura das variedades de Pimentão, Avelar e Ikeda. (Média de 4 repetições no mínimo).

\begin{tabular}{|c|c|c|c|c|c|c|c|c|}
\hline \multirow{2}{*}{$\begin{array}{c}\text { Idade } \\
\text { em } \\
\text { dias }\end{array}$} & \multicolumn{4}{|c|}{ Avelar } & \multicolumn{4}{|c|}{ Ikeda } \\
\hline & $\begin{array}{c}\text { mat. sêca } \\
\text { planta } \\
\text { inteira } \\
\text { (g) }\end{array}$ & $\begin{array}{c}\mathrm{n} \cdot{ }^{\circ} \\
\text { de } \\
\text { fôlhas }\end{array}$ & $\begin{array}{c}\mathrm{n} .{ }^{\circ} \\
\text { de } \\
\text { frutos }\end{array}$ & $\begin{array}{l}\text { compri- } \\
\text { mento } \\
(\mathrm{cm})\end{array}$ & $\begin{array}{c}\text { mat. sêca } \\
\text { planta } \\
\text { inteira } \\
(\mathrm{g})\end{array}$ & $\begin{array}{c}\text { n.o } \\
\text { de } \\
\text { fôlhas }\end{array}$ & $\begin{array}{c}\mathrm{n} .{ }^{\circ} \\
\text { de } \\
\text { frutos }\end{array}$ & $\begin{array}{c}\text { altura } \\
\text { da } \\
\text { planta } \\
(\mathrm{cm})\end{array}$ \\
\hline 30 & 0,032 & 6 & - & 10,00 & 0,031 & 6 & - & 10,00 \\
\hline 40 & 0,127 & 10 & - & 14,00 & 0,110 & 8 & - & 13,00 \\
\hline 60 & 1,160 & 19 & - & 20,00 & 1,060 & 16 & - & 17,40 \\
\hline 75 & 4,900 & 49 & 1,1 & 31,00 & 4,230 & 33 & 1,0 & 25,50 \\
\hline 90 & 20,670 & 112 & 8,2 & 46,00 & 18,640 & 94 & 8,0 & 42,40 \\
\hline 100 & 31,420 & 133 & 11,5 & 50,00 & 28,750 & 132 & 11,2 & 46,00 \\
\hline 115 & 58,540 & 190 & 14,0 & 60,00 & 59,000 & 182 & 13,0 & 58,00 \\
\hline
\end{tabular}

Pelos dados de matéria sêca da planta inteira, é patente o crescimento lento até 75 dias. Entre 75-90 dias de idade há um aumento brusco, com uma média superior a $1 \mathrm{~g} /$ dia. Esse crescimento é crescente, atingindo no período de 100-115, quase $2 \mathrm{~g} /$ dia.

Observaram-se algumas diferenças no entanto insignificantes entre as duas variedades a partir da segunda amostragem (40 dias). Estas discrepâncias mostram tendência a se anularem no final do ciclo.

Em média nos primeiros 75 dias, o crescimento da matéria sêca é apenas $8,5 \%$ do total, intensificando-se ràpidamente com a frutificação. 


\section{Absorção de Nutrientes}

As tabelas II e III apresentam as quantidades totais dos nutrientes em mg, extraídos por uma planta (raiz, caule, fôlhas, flôres e frutos) das variedades Avelar e Ikeda, respectivamente.

TABELA II - Extração dos nutrientes, em mg por planta inteira da variedade Avelar, em função da idade (Média de 4 repetições, no mínimo).

\begin{tabular}{crrrrrr}
\hline $\begin{array}{c}\text { Idade da } \\
\text { planta (dias) }\end{array}$ & $\mathrm{N}$ & $\mathrm{P}$ & $\mathrm{K}$ & $\mathrm{Ca}$ & $\mathrm{Mg}$ & $\mathrm{S}$ \\
\cline { 2 - 7 } & & & $\mathrm{mg}$ & & & \\
\hline 30 & 0,7 & 0,1 & 2,5 & 0,2 & 0,2 & 0,1 \\
40 & 2,9 & 0,4 & 10,1 & 1,1 & 0,8 & 0,2 \\
60 & 45,6 & 4,8 & 72,1 & 18,5 & 7,2 & 5,3 \\
75 & 187,0 & 19,5 & 293,1 & 74,3 & 29,3 & 18,4 \\
90 & 725,0 & 72,8 & $1.186,9$ & 741,4 & 110,6 & 64,6 \\
100 & 960,0 & 110,8 & $1.705,8$ & $1.194,2$ & 162,7 & 95,3 \\
115 & $1.658,7$ & 158,5 & $2.717,1$ & $2.123,9$ & 274,4 & 166,9 \\
\hline
\end{tabular}

TABELA III - Extração dos nutrientes, em $\mathrm{mg}$ por planta inteira da var. Ikeda, em função da idade (Média de 4 repetições, no mínimo).

\begin{tabular}{crrrrrr}
\hline $\begin{array}{c}\text { Idade da } \\
\text { planta (dias) }\end{array}$ & $\mathrm{N}$ & $\mathrm{P}$ & $\mathrm{K}$ & $\mathrm{Ca}$ & $\mathrm{Mg}$ & $\mathrm{S}$ \\
\cline { 2 - 7 } 30 & & & $\mathrm{mg}$ & & & \\
\hline 40 & 0,7 & 0,1 & 2,5 & 0,2 & 0,2 & 0,1 \\
60 & 2,9 & 0,4 & 2,5 & 1,0 & 0,7 & 0,2 \\
75 & 40,6 & 4,4 & 63,8 & 15,3 & 6,6 & 4,4 \\
90 & 159,5 & 16,5 & 239,2 & 61,8 & 24,0 & 14,4 \\
100 & 663,0 & 61,1 & $1.033,3$ & 658,2 & 103,7 & 54,1 \\
115 & 896,9 & 103,5 & $1.620,3$ & 987,5 & 155,2 & 97,7 \\
& $1.617,0$ & 146,7 & $2.776,3$ & $2.025,3$ & 263,7 & 181,6 \\
& & & & & &
\end{tabular}

E notório a pequena extração de nutrientes, até os 75 dias. A partir dêsse período, coincidindo com a crescente frutificação, ocorre um aumento espetacular na absorção, sempre crescente. Estas observações concordam com as obtidas por HAAG et al (1970), na var. Casca Dura. 
Aspecto muito interessante é a extração de cálcio. Há uma intensificação crescente e constante, chegando mesmo a ultrapassar a absorção de nitrogênio, após os 100 dias de idade. Devendo-se lembrar ser o solo de $\mathrm{pH}=6,5$, normal para a cultura do pimentão, não tendo sido necessário aplicação de calcário ou outro corretivo. Também interessante, é a diminuta extração de fósforo, inferior aos demais nutrientes.

Entre ambas as variedades são insignificantes as diferenças no início e no final do ciclo, tendendo a se anularem. Em tôrno de 75 dias, são um pouco maiores essas diferenças, extraindo a variedade Avelar mais que a var. Ikeda: $14,7 \% \mathrm{~N}, 15,4 \% \mathrm{P}, 18,4 \% \mathrm{~K}, 16,9 \% \mathrm{Ca}$, $18,1 \% \mathrm{Mg}$ e $21,8 \% \mathrm{~S}$, conforme dados na tabela IV. Observa-se ainda, um grande aumento na extração de S, pela var. Ikeda, no final do ciclo, ultrapassando um pouco a var. Avelar.

TABELA IV - Diferenças de absorção da var. Avelar sôbre a Ikeda, em porcentagens

\begin{tabular}{crrrrrr}
\hline $\begin{array}{c}\text { Idade em } \\
\text { dias }\end{array}$ & $\mathrm{N}$ & $\mathrm{P}$ & $\mathrm{K}$ & $\mathrm{Ca}$ & $\mathrm{Mg}$ & $\mathrm{S}$ \\
\cline { 2 - 7 } & & \multicolumn{7}{c}{$\%$} & & & \\
\hline \hline 30 & 0,0 & 0,0 & 0,0 & 0,0 & 0,0 & 0,0 \\
40 & 0,0 & 0,0 & 15,9 & 9,1 & 12,5 & 0,0 \\
60 & 10,7 & 8,3 & 11,6 & 17,3 & 8,4 & 17,0 \\
75 & 14,7 & 15,4 & 18,4 & 16,9 & 18,1 & 21,8 \\
90 & 8,6 & 16,1 & 12,5 & 11,3 & 6,3 & 16,3 \\
100 & 6,6 & 6,6 & 5,1 & 17,3 & 4,7 & $-3,9$ \\
110 & 2,6 & 7,5 & $-2,1$ & 4,7 & 3,9 & $-8,1$ \\
\hline
\end{tabular}

Com dados médios das tabelas II e III, verifica-se que uma planta de pimentão, cultura nas condições de campo em que o presente ensaio foi conduzido, absorve: $1,637,8 \mathrm{mg}$ de $\mathrm{N}, 152,6 \mathrm{mg} / \mathrm{P}$, $2.746,7 \mathrm{mg} / \mathrm{K} 2.074,6 \mathrm{mg} / \mathrm{Ca}, 269,0 \mathrm{mg} / \mathrm{Mg}$ e $174,2 \mathrm{mg} / \mathrm{S}$.

\section{CONCLUSÕES}

a) - crescimento: em ambas as variedades, é lento até os 75 dias, intensificando-se após este período, até o final do ciclo. Não há diferença significante entre elas.

b) - absorção de nutrientes : em geral é pequena a absorção de nutrientes, antes da frutificação (75 dias), aumentando bruscamente após este período. Entre as var. Avelar e Ikeda não ocorre diferença 
significante no início e no total dos nutrientes absorvidos no final. Porém, aos 75 dias de idade, uma planta da var. Avelar absorve mais que uma da Ikeda: $14,7 \% \mathrm{~N}, 15,4 \% \mathrm{P}, 18,4 \% \mathrm{~K}, 16,9 \% \mathrm{Ca}, 18,1 \% \mathrm{Mg}$ e $21,8 \% \mathrm{~S}$.

c) - com dados médios das duas variedades, nas condições do presente ensaio, numa cultura de pimentão abśorve em um hectare (25.000 plantas): 40,9 kg/N, 3,8 kg/P, 68,6 kg/K, 51,8 kg/Ca, 6,7 $\mathrm{kg} / \mathrm{Mg}$ e $4,3 \mathrm{~kg} / \mathrm{S}$.

\section{MINERAL NUTRITION OF VEGETABLES CROPS}

\section{SUMMARY}

XV. Studies on the mineral nutrition on two varieties of Sweet Pepper under field conditions.

Sweet pepper (Capsicum annum L.) varieties Avelar and Ikeda were cultivated under field conditions: Periodically, from 30 th day, plants were harvested and analysed for $\mathrm{N}, \mathrm{P}, \mathrm{K}, \mathrm{Ca}, \mathrm{Mg}$ and $\mathrm{S}$.

Data obtained allowed the following conclusions:

a) the initial rate of growth of the sweet pepper during the first 75 days is rather slow. There is no significant growth difference between those varieties.

b) - the uptake of macronutrients is small until the 75 days, increating with the fructifcation. There is no significant difference between the varieties, in the total of nutrients absorved. At the 75 days old a plant of var. Avelar absorbs, more than Ikeda plant: $14.7 \% \mathrm{~N}, 15.4 \% \mathrm{P} 18.4 \% \mathrm{~K}, 16.4 \% \mathrm{Ca}, 18.1 \% \mathrm{Mg}$ and $21.8 \% \mathrm{~S}$.

c) - the amounts of elements in $\mathrm{kg} / \mathrm{ha}$, absorved by a population of 25,000 plants under the field conditions of these experiment, were: $40.9 \mathrm{~kg} / \mathrm{N}, 3.8 \mathrm{~kg} / \mathrm{P}, 68.6 \mathrm{~kg} / \mathrm{K}, 51.8 \mathrm{~kg} / \mathrm{Ca}, 6.7 \mathrm{~kg} / \mathrm{Mg}, 4.3 \mathrm{~kg} / \mathrm{S}$.

\section{LITERATURA CITADA}

BERNARDI, J. B. 1962. Adubação para pimentação. O Agrônomico. Campinas S.P. 14(11-12):8-9.

CATANI, R. A.; J. R. GALLO \& H. GARGANTINI - 1955. Amostragem de Solo. Métodos de análise, Interpretação e Indicações Gerais para fins de fertilidade Bol. n. ${ }^{\circ}$ 69. Inst. Agron. Campinas. Campinas E.S.P.

HAAG, H. P., P HOMA \& T. KIMOTO. 1970. Nutrição Mineral de Hortaliças. V. Absorção de nutrientes pela cultura do pimentão. O Solo. LXII (2):7-11.

LOTT, W. L.; J. P. NERY; J. R. GALLO \& J. C. MEDCALF. 1956. A técnica de Análise Foliar aplicada ao cafeeiro. Bol. n. ${ }^{\circ}$ 79. Inst. Agron. Campinas Campinas - E. S. Paulo.

MALAVOLTA, E. 1957. Práticas de Química Orgânica e Biológica. C. A. "Luiz de Queiroz". Piracicaba - S.P. 
RANZANI, G., O. FREIRE \& T. KINJO. 1966. Carta de Solos do Município de Piracicaba. Centro de Estudos de Solos. E.S.A. "Luiz de Queiroz". Piracicaba - E.S.P.

THE PERKIN ELMER CORP. 1966. Analytical Methods for Atomic Absorption Spectrophotometry. Perkin-Elmer Corp. Connecticut.

TOTH, S. J., A. L. PRINCE; A. WALLACE, \& D. S. MIKKELSEN. 1948. Rapid Quantitative Determination of Eight Mineral Elements in Plant Tissues by a Sistematic Procedure Envolving the Use Flame Photometer. Soil Sci. $66: 459-466$. 
